\title{
Role of core polarization curvature of screw dislocations in determining the Peierls stress in bcc Ta: A criterion for designing high-performance materials
}

\author{
Guofeng Wang, ${ }^{*}$ Alejandro Strachan, ${ }^{\dagger}$ Tahir Çăğın, and William A. Goddard III ${ }^{\ddagger}$ \\ Materials and Process Simulation Center, Beckman Institute (139-74), California Institute of Technology, Pasadena, California 91125
}

(Received 21 January 2003; published 28 April 2003)

\begin{abstract}
We use a family of embedded atom model potentials all based on accurate quantum-mechanical calculations to study the relation between Peierls stress and core properties of the $1 / 2 a\langle 111\rangle$ screw dislocation in bcc tantalum (Ta). We find that the Peierls stress $\left(\sigma_{P}\right)$ is a function of the core-polarization curvature $(\Pi)$ near the equilibrium core configuration. Our results suggest that the computationally available quantity $\Pi$ is a useful criterion for designing high-performance materials.
\end{abstract}

DOI: 10.1103/PhysRevB.67.140101

PACS number(s): 62.20.Fe, 61.72.Lk

Determining the fundamental atomistic mechanisms that underlie plastic deformations of macroscopic materials is a key, enabling step toward designing materials with improved and tailored properties. This is particularly important for bcc metals, which are the basis of some of the highestperforming alloys, but whose behavior is more complex than fcc and hep materials. ${ }^{1}$ Computer simulations could be used to investigate the individual and collective dislocation motions in these materials and guide the development of highperformance materials. $^{2}$

At low temperatures, plasticity in bcc metals is governed by low mobility screw dislocations with Burgers vector $\mathbf{b}$ $=1 / 2 a\langle 111\rangle$. It is generally believed that the core structure of these dislocations is a controlling factor of their mobility. ${ }^{3}$ Theoretical studies on these dislocations have led to two types of core structures: asymmetric ${ }^{3-5}$ core and symmetric core ${ }^{5-8}$ In differential displacement (DD) maps, ${ }^{3}$ the asymmetric core [Fig. 1(a)] spreads in three $\langle 112\rangle$ directions on $\{110\}$ planes, while the symmetric core [Fig. 1(b)] is compact. The main differences between these two types of cores are the relative displacements in the $\langle 111\rangle$ direction of the two sets of three atoms in the core [atoms $\{A, C, E\}$ and $\{B$, $D, F\}$ in Fig. 1(c)]. The "polarization" 9 of the dislocation core can be used to distinguish different core configurations and is defined by Eq. (1),

$$
p=\frac{\left|d_{B C}-d_{A B}\right|+\left|d_{D E}-d_{C D}\right|+\left|d_{F A}-d_{E F}\right|}{b},
$$

where, $d_{X Y}(X, Y=A, B, C, D, E$, or $F)$ is the relative displacement between two neighboring atoms in the two columns denoted as $X$ and $Y$ in Fig. 1(c), and $b$ is the magnitude of the dislocation Burgers vector. Thus a symmetric core leads to $p=0$, while $p=1$ corresponds to a fully asymmetric core.

The Peierls stress is the minimal shear stress required to move a dislocation in an otherwise perfect crystal. In bcc metals, the Peierls stress depends strongly on the orientation of the shearing. ${ }^{3,5}$ In this paper, we study in detail the twinning $\left(\chi=-30^{\circ}\right)$ and antitwinning $\left(\chi=30^{\circ}\right)$ shearing on (112) planes, where $\chi$ is the angle between the plane with the maximum shear stress and the neighboring (110) plane. Two recent atomistic calculations ${ }^{8,10}$ of the Peierls stress for Ta show good agreement when the applied stress is in the twinning direction: $a b$ initio density-functional theory (DFT) cal- culations lead to $675 \mathrm{MPa}$ and calculations using the model generalized pseudopotential theory (MGPT) potential lead to $600 \mathrm{MPa}$. However, for the antitwinning direction the calculated Peierls stresses differ by a factor of 2.6 [3.60 GPa from $a b$ initio calculations and $1.40 \mathrm{GPa}$ using the MGPT force field (FF)]. This large difference is obtained although both calculations lead to symmetric dislocation cores with zero or very small ( $p=0.004$ in Ref. 8 ) polarization. It implies that the equilibrium dislocation core structure by itself does not simply determine the Peierls stress. This motivated our systematic examination of the relation between dislocation core properties and Peierls stress.

To explore the relationship between dislocation core properties and Peierls stress, we developed a family of firstprinciples-based embedded atom model (EAM) force fields denoted as $q \mathrm{EAM} i(i=1-4)$ for Ta, each of which closely matches a number of properties from the quantummechanical (QM) (generalized gradient approximation DFT) calculations (see details in Ref. 11). Table I shows that the four $q$ EAM FF's lead to the similar lattice parameters and elastic constants for bcc Ta at $0 \mathrm{~K}$. Moreover, all four $q \mathrm{EAM}$ FF's predict similar $\gamma$ surfaces (the energy profile for a generalized stacking fault between two semi-infinite half crystals first displaced relative to each other by a vector on a crystallographic plane then relaxed only in the direction perpendicular to the plane). This is shown in Fig. 2 for the $\langle 111\rangle$ direction in the $\{112\}$ plane, which qualitatively reveals the twinning and the antitwinning asymmetry. Our $q$ EAM FF results agree well with the $a b$ initio and MGPT data. ${ }^{8}$ However, the MGPT FF (Ref. 8) and all $q$ EAM FF's lead to an asymmetry in the $\gamma$ surface much smaller than that of the $a b$ initio calculation.

The main difference among the $q$ EAM FF's is in polarization of the screw dislocation core. We deliberately constrained the $q$ EAM FF's to provide a range of different dislocation core-polarization behaviors: (i) $q$ EAM1 leads to an asymmetric core, with the DD map in Fig. 1(a) and the relaxation map in Fig. 1(c). The equilibrium dislocation core has a polarization of 0.81 . (ii) $q$ EAM 2 is adjusted to have a dislocation core energy nearly independent of polarization. (iii) $q$ EAM3 is adjusted to predict a symmetric dislocation core but with the core-polarization curvature (second derivative of core energy with respect to polarization) around the equilibrium core configuration similar to the $q \mathrm{EAM} 1$. (iv) 


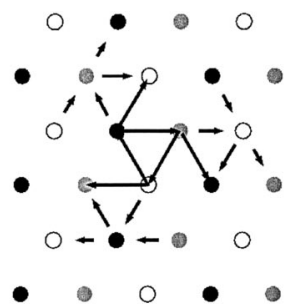

(a)

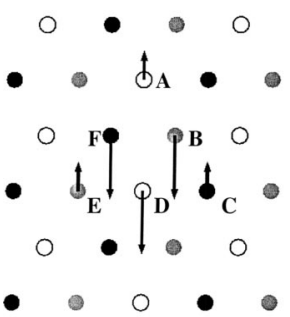

(c)

FIG. 1. The DD maps for (a) the asymmetric core (from $q$ EAM1) and (b) the symmetric core (from $q$ EAM4) of screw dislocations in bcc Ta. In DD maps, the atoms (circles) are projected on a (111) plane; the arrows indicate the relative displacements of neighboring atoms in the [111] direction with respect to their positions in the perfect bcc crystal. When an arrow spans the full distance between two atoms, the relative displacement is $b / 3$. For clarity, the relative displacements less than $b / 12$ are not shown in the above two figures. (c) The relaxation map for the same asymmetric core as in (a). In the figure, the arrows indicate that after relaxation from continuum elasticity theory predictions the three central columns (columns B, D, and F) of atoms translate simultaneously by $0.267 \AA$ in the $[\overline{1} \overline{1} \overline{1}]$ direction while atoms in columns A, C, and E translate by $0.123 \AA$ in the [111] direction. The relaxations of other atoms are less than $0.05 \AA$ and are not shown.

$q$ EAM4 leads to a symmetric core with an energy dependence on polarization very similar to that from our $a b$ initio calculation.

Figure 3 shows the relative energy (the energy difference between the polarized cores and the zero-polarization symmetric core) as a function of polarization for the various

TABLE I. Experimental and theoretical values of lattice parameter $a(\AA)$; elastic moduli $C_{11}(\mathrm{GPa}), C_{12}(\mathrm{GPa})$, and $C_{44}(\mathrm{GPa})$; and the shear modulus in the $\langle 111\rangle$ direction $G(\mathrm{GPa})\left[G=\left(C_{11}-C_{12}\right.\right.$ $\left.+C_{44}\right) / 3$ ] for bcc Ta from our $q$ EAM force fields, the MGPT FF, $a b$ initio calculation, and experiments.

\begin{tabular}{lccccc}
\hline \hline & $a$ & $C_{11}$ & $C_{12}$ & $C_{44}$ & $G$ \\
\hline$q$ EAM1 & 3.32 & 273 & 138 & 69.6 & 68.2 \\
$q$ EAM2 & 3.33 & 254 & 155 & 67.4 & 55.5 \\
$q$ EAM3 & 3.35 & 255 & 148 & 60.2 & 55.7 \\
$q$ EAM4 & 3.32 & 257 & 148 & 77.3 & 62.1 \\
MGPT $^{\mathrm{a}}$ & 3.30 & 266 & 161 & 82.5 & 62.5 \\
ab $_{\text {initio }}{ }^{\mathrm{b}}$ & 3.23 & 291 & 175 & 53.0 & 56.3 \\
Experiment $^{\mathrm{c}}$ & 3.30 & 266 & 158 & 87.4 & 65.1 \\
\hline \hline
\end{tabular}

${ }^{\mathrm{a}}$ Reference 8 .

${ }^{\mathrm{b}}$ Reference 10 .

${ }^{\mathrm{c}}$ Reference 12 .

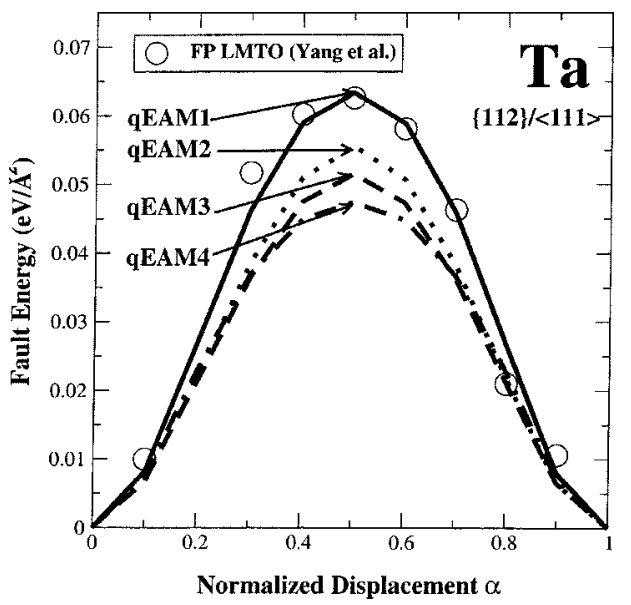

FIG. 2. The $\langle 111\rangle$ line in the $\{112\}$ plane $\gamma$ surface for bcc Ta as calculated with the $q$ EAM potentials and the $a b$ initio method. In the $q$ EAM calculations, fixed boundary conditions are applied after 48 atomic planes on both sides of the faulted surface. The atoms are relaxed in the [112] direction to the full convergence that the force on each atoms is no more than $3.5 \times 10^{-4} \mathrm{eV} / \AA$.

$q$ EAM FF's. In order to obtain the energies for the nonequilibrium core configurations, we fixed the positions of the six atoms [atoms $\mathrm{A}-\mathrm{F}$ in Fig. 1(c)] in the dislocation direction and optimized the energy for the model system. In all calculations, we used a periodic simulation cell with parameters of $\mathbf{X}=3 a[11 \overline{2}], \quad \mathbf{Y}=5 a[1 \overline{1} 0]$, and $\mathbf{Z}=1 / 2 a[111]$ (90 atoms per cell) and the dislocation quadrupole arrangement.

To obtain the QM results in Fig. 3 (open circles), we evaluated the energies using density-functional theory (DFT) with the local-density approximation ${ }^{13,14}$ (LDA) for the relaxed atomic configurations obtained with $q$ EAM1. We used the Hamann-type generalized norm-conserving pseudopotential for Ta with the nonlinear core correction. ${ }^{15,16}$ We used eight $k$ points in the direction of the dislocation line and one $k$ point in the normal directions. These QM calculations pre-

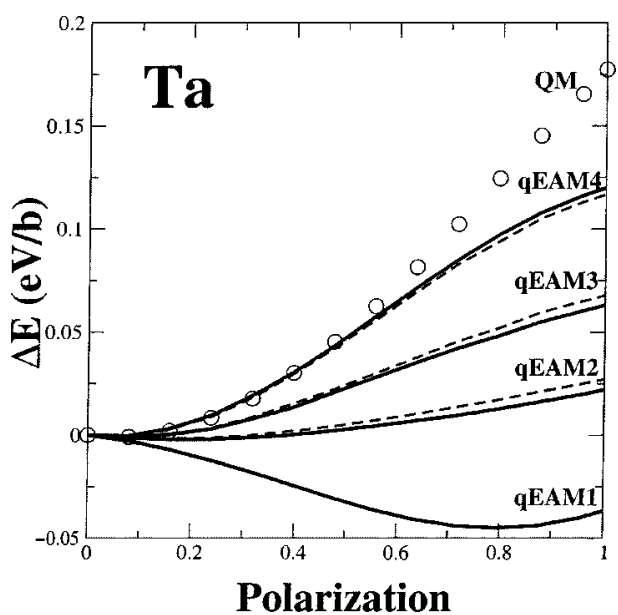

FIG. 3. The dependence of the dislocation core energy with its polarization from our $q$ EAM FF's and from DFT-LDA calculations. The solid lines in the figure show the energy difference for the relaxed structures using the $q$ EAM FF's, while the dashed lines show the results from the energy evaluation of the relaxed structures from the $q$ EAM1 FF. The QM results are shown as circles. 
TABLE II. The calculated core energy (eV/b) and Peierls stresses (in units of shear modulus $G$ ) for $1 / 2 a\langle 111\rangle$ screw dislocations in bcc Ta using our $q \mathrm{EAM}$ force fields.

\begin{tabular}{ccccccccc}
\hline \hline & \multicolumn{2}{c}{ Core energy } & & \multicolumn{2}{c}{ Peierls stress (twinning) } & & \multicolumn{2}{c}{ Peierls stress (antitwinning) } \\
\cline { 2 - 3 } \cline { 7 - 8 } Force field & $r_{c}=2 b$ & $r_{c}=1.75 b$ & & $9 \times 15$ & $13 \times 21$ & & $9 \times 15$ & $13 \times 21$ \\
\hline$q$ EAM1 & 1.297 & 1.190 & & 0.0067 & 0.0085 & & 0.0152 & 0.0170 \\
$q$ EAM2 & 1.154 & 1.065 & & 0.0004 & 0.0011 & & 0.0022 & 0.0040 \\
$q$ EAM3 & 1.147 & 1.054 & & 0.0057 & 0.0065 & & 0.0108 & 0.0108 \\
$q$ EAM4 & 1.161 & 1.063 & & 0.0126 & 0.0132 & & 0.0496 & 0.0512 \\
\hline \hline
\end{tabular}

dict the symmetric core with the lowest energy, consistent with the previous $a b$ initio results from direct minimization. 6,7 Although we did not optimize the atomic configurations in the QM calculations, the small change in relative energies (comparing solid and dashed lines in Fig. 3) indicates that a full optimization would not change the conclusions.

We calculated the core energy of $1 / 2 a\langle 111\rangle$ screw dislocations using these $q$ EAM FF's and the same procedure described in Ref. 17. In these calculations, we used quadrupole arrays of dislocations with system sizes ranging from 1890 to 5670 atoms and optimized all atomic coordinates using the various $q$ EAM FF's. It should be mentioned that it is possible to reduce the computational effort here by using a special shape of the supercell proposed in Ref. 18 containing only half the simulation volume. Since the computational costs with our force fields are not significant, we chose to use the simple orthorhombic quadruple cells.

Table II shows the calculated screw dislocation core energies using core radii of $r_{c}=2 b$ and $r_{c}=1.75 b$. All four $q$ EAM FF's lead to larger values than the $a b$ initio calculation $^{6}\left(E_{c}=0.86 \mathrm{eV} / \mathrm{b}\right.$ for $\left.r_{c}=2 b\right)$ and the MGPT FF calculation $^{8}\left(E_{c}=0.60 \mathrm{eV} / \mathrm{b}\right.$ for $\left.r_{c}=1.75 b\right)$, but the core energies for the symmetric core systems ( $q$ EAM2, $q$ EAM3, and $q$ EAM4) are very close to each other and averaged to $1.154\left(r_{c}=2 b\right)$ and $1.061 \mathrm{eV} / \mathrm{b}\left(r_{c}=1.75 b\right)$. The asymmetric core $(q$ EAM1) only leads to slightly higher energy, 1.297 $\left(r_{c}=2 b\right)$ and $1.190 \mathrm{eV} / \mathrm{b}\left(r_{c}=1.75 b\right)$.

We determined the Peierls stresses $\sigma_{p}^{\text {twin }}$ (twinning) and $\sigma_{p}^{\text {anti }}$ (antitwinning) by applying pure shear stress on simulation cells containing a $[1 \overline{1} 0]$ screw dislocation dipole (two dislocations with opposite Burgers vectors aligned in the [1ํㅣ direction). To obtain the initial zero stress configurations we minimized the total energy by relaxing atomic positions and cell parameters. We then applied finite shear stresses in increments of $20 \mathrm{MPa}$ until the dislocations moved. For each stress state, we performed 10 ps of NPT molecular-dynamics (MD) simulation followed by 25 ps of NVT MD simulation at $0.001 \mathrm{~K}$. To check the size convergence of our calculation, we carried out simulations for two different cell sizes: a $9 \times 15$ cell $(\mathbf{X}=9 a[11 \overline{2}], \quad \mathbf{Y}$ $=15 a[\overline{1} 10]$, and $\mathbf{Z}=7 a / 2[111])$ with 5670 atoms, and a $13 \times 21 \quad$ cell $\quad(\mathbf{X}=13 a[11 \overline{2}], \quad \mathbf{Y}=21 a[\overline{1} 10], \quad$ and $\quad \mathbf{Z}$ $=7 a / 2[111]$ ) with 11466 atoms. Table II reports the ratio of the calculated Peierls stresses to the $\langle 111\rangle$ shear stress $G$ (see Table I).
Important points here are (i) the Peierls stresses differ dramatically for $q \mathrm{EAM} 2, q \mathrm{EAM} 3$, and $q \mathrm{EAM} 4$, each of which leads to symmetric core structure and similar core energy. The main difference in these potentials is the corepolarization curvature. (ii) The $q$ EAM1 and $q$ EAM3 lead to completely different equilibrium core structures (asymmetric versus symmetric), but predict similar Peierls stresses. As pointed out before, they have similar values for corepolarization curvature. (iii) The $\gamma$ surface from the $q$ EAM1 agrees best with the $a b$ initio results, but the $q$ EAM1 leads to an asymmetric core and much different Peierls stresses than the $a b$ initio calculations. (iv) Although the $q$ EAM2 leads to a $\gamma$ surface agreeing better with the $a b$ initio results than the $q$ EAM4, the $q$ EAM4 predicts Peierls stresses much closer to the $a b$ initio calculation.

The above results [(i) and (ii)] indicate that such equilibrium dislocation core properties as structure, polarization, and energy do not correlate with Peierls stress. Findings (iii) and (iv) suggest that the $\gamma$ surface is not an important factor in modeling bcc screw dislocation behavior, which contrasts with other suggestions in the literature (for instance, Ref. 3).

Our studies indicate that it is the core-polarization curva-

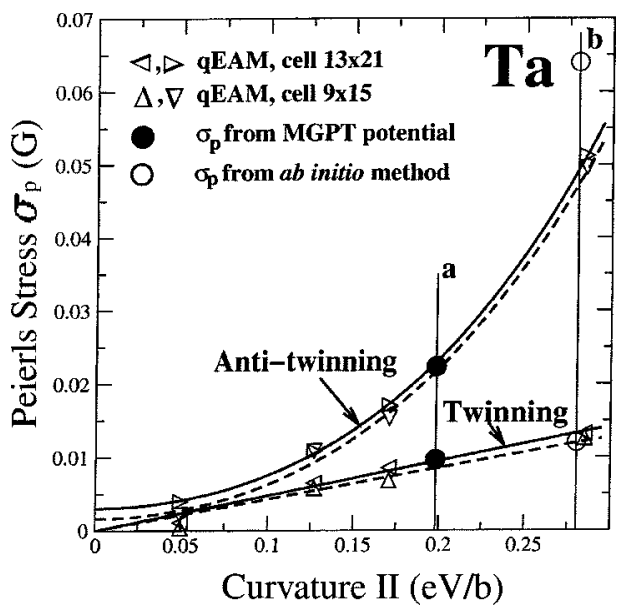

FIG. 4. The Peierls stress as a function of core-polarization curvature $\Pi$. The solid curves show the best fits of the results for the cell size $13 \times 21$, while the dashed curves are for the cell size 9 $\times 15$. For solid curves, the fitted linear function for the twinning shear is $\sigma_{p}=0.0472 * \Pi\left(R^{2}=0.8552\right)$ and the fitting function for the antitwinning shear is $\sigma_{p}^{\mathrm{anti}}=(2.6492) \cdot \Pi^{4}+(0.4259) \cdot \Pi^{2}$ $+0.0030\left(R^{2}=0.9998\right)$. The corresponding $\Pi$ is 0.198 for the vertical line "a" showing the results from the MGPT FF and 0.2804 for the vertical line "b" describing the QM results. 
ture $\Pi$ that is responsible for the dramatic dependence of Peierls stress on various potentials. This is seen in Fig. 4, which shows the twinning and antitwinning Peierls stresses as a function of $\Pi$ for the various $q$ EAM FF's. In Fig. 4, the empty triangles represent the results from different potentials. This correlation is plausible because we find that (i) the polarization of an asymmetric core decreases first before its translation, consistent with the microscopic picture proposed by Hirsch $^{19}$ and (ii) the polarization of a symmetric core increases before it moves, which has been observed previously in Ref. 20.

Contrary to the belief that a symmetric core would not change its polarization during its motion, our simulations show a symmetric dislocation core under shear stress first extends in three $\langle 112\rangle$ directions on $\{110\}$ planes and transforms into the asymmetric (polarized) core before it moves. After translating one step, the polarized core transforms back toward the zero-polarized symmetric core. Thus, regardless of the core structure (symmetric or asymmetric) the Peierls stress required to move the dislocation depends on the average energy variation over a range of polarizations. We find (Fig. 4) that the Peirls stress changes monotonically with $\Pi$.

Figure 4 shows that $\sigma_{p}^{\text {twin }}$ varies more slowly with $\Pi$ than does $\sigma_{p}^{\text {anti }}$. Consequently $\sigma_{p}^{\text {twin }}$ is less sensitive to differences in $\Pi$ than is $\sigma_{p}^{\text {anti }}$. This explains why various Peierls stress calculations for Ta (Refs. 8 and 10) agree in the twinning direction but disagree greatly in the antitwinning direction. This may also explain the observation in Ref. 10 of a good agreement for $\sigma_{p}^{\text {twin }}$ of Mo from different calculations but the large divergence of $\sigma_{p}^{\text {anti }}$.

To gauge the reliability of the correlation in Fig. 4, we predicted the $a b$ initio Peierls stresses using the curvature $\Pi=0.2806$ derived from our QM polarization curve in Fig. 3. The predicted QM Peierls stresses are $\sigma_{p}^{\mathrm{twin}}=0.013 G$ and $\sigma_{p}^{\text {anti }}=0.049 G$ compared to the direct $\mathrm{QM}$ calculation results ${ }^{10}$ of $\sigma_{p}^{\text {twin }}=0.012 G$ and $\sigma_{p}^{\text {anti }}=0.064 G$. The agree- ment is fairly good. Moreover, the MGPT results appear also to be consistent with our model. If we assume $\Pi=0.198$, then the functions in Fig. 4 lead $\sigma_{p}^{\text {twin }}=0.00934 G$ and $\sigma_{p}^{\text {anti }}$ $=0.02290 G$ to within $3 \%$ of the MGPT calculations ${ }^{8}$ $\left(\sigma_{p}^{\text {twin }}=0.00962 G\right.$ and $\left.\sigma_{p}^{\text {anti }}=0.02234 G\right)$.

Thus, we conclude that the dominant factor underlying the magnitude of the Peierls stress in bcc Ta is the corepolarization curvature $\Pi$. This dependence arises because a change in core polarization occurs as a dislocation (regardless of core structure) migrates from one equilibrium site to the next. Two additional observations support the above conclusion.

(i) The calculated Peierls stresses from the $q$ EAM2 FF are significantly lower than previous calculations, $8,10,20$ which are at least two or three times larger than the experimental data. The only significant difference among $q$ EAM2 with the other potentials is just the dislocation corepolarization curvature.

(ii) In addition to $\sigma_{p}^{\text {twin }}$ and $\sigma_{p}^{\text {anti }}$, we also calculated the Peierls stress $\sigma_{p}^{\{110\}}$ whose maximum resolved shear stress plane is in the $\{110\}$ plane. Our results for $\sigma_{p}^{\{110\}}, 0.0117 G$ $(q$ EAM $1, \Pi=0.171), \quad 0.0022 G \quad(q$ EAM $2, \Pi=0.050)$, $0.0068 G(q$ EAM3,$\Pi=0.127)$, and $0.0138 G(q$ EAM4, $\Pi$ $=0.285$ ), are consistent with the conclusion that Peierls stress correlates with $\Pi$.

This paper reports a direct relation between the dislocation core-polarization curvature and Peierls stress. It provides a new view of plastic deformation of bcc metals, suggesting a criterion for designing materials with improved plasticity. Our results suggest that a bcc material with a given set of elastic constants could be made more ductile by minimizing $\Pi$ and made into a higher flow stress material by maximizing I. $^{21}$

We thank Peter A. Schultz of Sandia National Lab for his help with the QM calculations. This research was supported by Caltech Project No. DoE-ASC-ASAP.
*Present address: Materials Science Division, Lawrence Berkeley National Laboratory, Berkeley, CA 94720.

†Present address: Los Alamos National Laboratory, Los Alamos, NM 87545.

¥Author to whom correspondence should be addressed. Email address: wag@wag.caltech.edu

${ }^{1}$ J. W. Christian, Metall. Trans. A 14A, 1237 (1983).

${ }^{2}$ V. V. Bulatov et al., Nature (London) 391, 669 (1998).

${ }^{3}$ V. Vitek, Cryst. Lattice Defects 5, 1 (1974).

${ }^{4}$ W. Xu and J. A. Moriarty, Phys. Rev. B 54, 6941 (1996).

${ }^{5}$ M. S. Duesbery and V. Vitek, Acta Mater. 46, 1481 (1998).

${ }^{6}$ S. Ismail-Beigi and T. A. Arias, Phys. Rev. Lett. 84, 1499 (2000).

${ }^{7}$ C. Woodward and S. I. Rao, Philos. Mag. A 81, 1305 (2001).

${ }^{8}$ L. H. Yang, P. Söderlind, and J. A. Moriarty, Philos. Mag. A 81, 1355 (2001).

${ }^{9}$ A. Seeger and C. Wuthrich, Nuovo Cimento Soc. Ital. Fis., B 33B, 38 (1976).

${ }^{10}$ C. Woodward and S. I. Rao, Phys. Rev. Lett. 88, 216402 (2002).

${ }^{11}$ A. Strachan, T. Çăgın, O. Gülseren, S. Mukherjee, R. E. Cohen, and W. A. Goddard, cond-mat/0208027 (unpublished).

${ }^{12}$ Single Crystal Elastic Constants and Calculated Aggregate Prop- erties: A Handbook, edited by G. Simmons and H. Wang (MIT, Cambridge, MA, 1971).

${ }^{13}$ J. Perdew and A. Zunger, Phys. Rev. B 23, 5048 (1981).

${ }^{14}$ M. Ceperley and B. J. Alder, Phys. Rev. Lett. 45, 566 (1980).

${ }^{15}$ D. R. Hamann, Phys. Rev. B 40, 2980 (1989).

${ }^{16}$ S. G. Louie, S. Froyen, and M. L. Cohen, Phys. Rev. B 26, 1738 (1982).

${ }^{17}$ G. Wang, A. Strachan, T. Çağin, and W. A. Goddard, Mater. Sci. Eng., A 309-310, 133 (2001).

${ }^{18}$ J. R. K. Bigger et al., Phys. Rev. Lett. 69, 2224 (1992).

${ }^{19}$ P. B. Hirsch, in Proceedings of the Fifth International Conference on Crystallography (Cambridge University, Cambridge, England, 1960), p. 139.

${ }^{20}$ K. Ito and V. Vitek, Philos. Mag. A 81, 1387 (2001).

${ }^{21}$ See EPAPS Document No. E-PRBMDO-67-R02314 for eight additional tables and a supplemental figure. A direct link to this document may be found in the online article's HTML reference section. The document may also be reached via the EPAPS homepage (http://www.aip.org/pubservs/epaps.html) or from ftp.aip.org in the directory /epaps/. See the EPAPS homepage for more information. 